\title{
BMJ Open Global estimate of the prevalence of post-traumatic stress disorder among adults living with HIV: a systematic review and meta-analysis
}

Chulei Tang (D) , ${ }^{1}$ Lloyd Goldsamt, ${ }^{2}$ Jingjing Meng, ${ }^{1}$ Xueling Xiao, ${ }^{1}$ Li Zhang, ${ }^{2}$ Ann Bartley Williams, ${ }^{1}$ Honghong Wang ${ }^{1}$

To cite: Tang C, Goldsamt L, Meng J, et al. Global estimate of the prevalence of posttraumatic stress disorder among adults living with HIV: a systematic review and meta-analysis. BMJ Open 2020;10:e032435. doi:10.1136/ bmjopen-2019-032435

- Prepublication history and additional material for this paper are available online. To view these files, please visit the journal online (http://dx.doi. org/10.1136/bmjopen-2019032435).

Received 20 June 2019 Revised 29 February 2020 Accepted 03 March 2020

\section{Check for updates}

(c) Author(s) (or their employer(s)) 2020. Re-use permitted under CC BY-NC. No commercial re-use. See rights and permissions. Published by BMJ.

${ }^{1}$ Xiangya Nursing School, Central South University, Changsha, Hunan, China

${ }^{2}$ New York University Rory Meyers College of Nursing, New York, New York, USA

Correspondence to Professor Honghong Wang; honghong_wang@hotmail.com

\section{ABSTRACT}

Objectives Although people living with HIV (PLWH) have been disproportionately affected by post-traumatic stress disorder (PTSD), the global prevalence of PTSD among PLWH is unknown. This study aimed to systematically review the prevalence of PTSD among PLWH worldwide and explore variation in prevalence across sociodemographic and methodological factors. Design A meta-analysis using a random-effects model was conducted to pool the prevalence estimated from individual studies, and subgroup analyses were used to analyse heterogeneities.

Setting, participants and measures Observational studies providing PTSD prevalence data in an adult HIV population were searched from January 2000 to November 2019. Measurements were not restricted, although the definition of PTSD had to align with the Diagnostic and Statistical Manual of Mental Disorders or the International Classification of Diseases diagnostic criteria.

Results A total of 38 articles were included among 2406 records identified initially. The estimated global prevalence of PTSD in PLWH was $28 \%$ (95\% Cl $24 \%$ to $33 \%$ ). Significant heterogeneity was detected in the proportion of PLWH who reported PTSD across studies, which was partially explained by geographic area, population group, measurement and sampling method $(p<0.05)$.

Conclusion PTSD among PLWH is common worldwide. This review highlights that PTSD should be routinely screened for and that more effective prevention strategies and treatment packages targeting PTSD are needed in PLWH.

\section{INTRODUCTION}

Globally, there were $>36$ million people living with HIV (PLWH) by the end of $2017 .{ }^{1}$ Although increasingly expanded access to antiretroviral therapy (ART) has led to a prolonged life span, a large portion of PLWH still suffer a significant burden due to psychiatric disorders. ${ }^{23}$ Post-traumatic stress disorder (PTSD) is a mental health condition following exposure to a life-threatening event, or extreme or repeated exposure to traumatic events. ${ }^{4}$ A growing body of
Strengths and limitations of this study

- This is the first meta-analysis providing comprehensive assessment on the prevalence of post-traumatic stress disorder (PTSD) among adults living with HIV.

- Several subgroup analyses were conducted to examine the influence of diverse sociodemographic backgrounds as well as methodological heterogeneity.

- This review was conducted with specific definitions of PTSD as part of the inclusion criteria to help in acquiring high level of evidence, which also limited the number of eligible studies.

- A lack of studies carried out on the topic in lowincome and middle-income countries could underestimate the burden of HIV-PTSD prevalence of the world.

- We did not undertake a search of grey literature, restricting our sample to articles published in peerreviewed journals.

literature indicates that PLWH have a heightened risk for PTSD. ${ }^{56}$ PLWH are more likely to report a history of traumatic and stressful life experiences than the general population, including childhood sexual/physical abuse and intimate partner violence. ${ }^{7-9}$ PTSD may precede an HIV-positive diagnosis due to previous traumatic experiences. In addition, being diagnosed with HIV, a life-threatening illness, is a potentially traumatic event in and of itself, and PTSD may emerge as a result of this diagnosis. Infection with HIV causes a progressive destruction of the immune system, which increases the susceptibility to malignancies that threaten a person's life, and being labelled and associated with HIV stigma, taking multiple daily medications and experiencing repeated unpleasant side effects constitute potentially traumatic events. ${ }^{10}$ These high rates of potential trauma exposure during the course of the illness may also increase the likelihood of developing PTSD. 
Generally, the concept of PTSD has been broadly applied to military veterans, survivors of disasters and accidents and victims of violent assaults. PTSD as a serious and costly health problem in the general population is well recognised, and impairs a person's physical health, and greatly influences social functioning. ${ }^{11}$ The co-occurrence of PTSD and HIV-infection creates even more challenges regarding both the treatment of PTSD and the management of HIV. PTSD can negatively impact medication adherence and impair immune functioning, ${ }^{12-15}$ both of which are especially critical for PLWH. PLWH with PTSD have been found to be at increased risk of somatisation and physical disorders. ${ }^{16}$ The illness experience of PTSD, such as persistent avoidance and re-experiencing the traumatic event, may also compromise quality of life and cause significant distress in PLWH. ${ }^{717}$ Subsequently, prolonged and untreated PTSD may lead to exacerbation of PTSD as well as HIV progression. ${ }^{18}$ There is also evidence suggesting that reductions in PTSD symptom severity are related to improved HIV-related outcomes. ${ }^{19}$ Given that PTSD elevates HIV risk behaviours ${ }^{20} 21$ and the possibility of HIV transmission also increases along with disease progression, PLWH suffering from PTSD are an important group to understand and with whom to intervene.

Valid data on PTSD prevalence among PLWH are essential. Evidence on the extent of PTSD among PLWH is needed to raise awareness and recommend clinical management. Although a number of studies have investigated the prevalence of PTSD in PLWH, findings have been inconsistent. ${ }^{22}$ While studies have reported varied incidence, few efforts have been made to aggregate existing research using meta-analysis techniques. There was one published meta-analysis estimating the pooled prevalence of PTSD among women living with HIV in $2012 .{ }^{23}$ But the lack of studies with other groups lowered the generalisability and global relevance of their findings. Moreover, the meta-analysis was conducted in 2012, and the increase of publications about PTSD among PLWH since then ${ }^{24-26}$ suggests that a timely update is urgently needed. Indeed, several reviews provide a useful overview of the possible prevalence of PTSD among $\mathrm{PLWH}^{22}{ }^{27}$; however, a number of gaps remain. First, to date, there has been no attempt to achieve a better understanding of the epidemiology of PTSD in PLWH and the reviews did not aim at an overall PTSD prevalence specifically. Second, economically and politically, while precise estimates of prevalence can support efficient allocation of resources, factors that might influence PTSD prevalence, such as population characteristics and sociocultural context have been neglected.

Therefore, to fill these knowledge gaps, we aimed to provide an estimate for the worldwide prevalence of PTSD in PLWH and to test whether PTSD prevalence in PLWH differs based between population groups, country income groups and study characteristics. This information can then be used to inform further practice and research for this highly comorbid group.
METHODS

\section{Search strategy}

Systematic searches were conducted through five electronic English databases (Medline, Web of Science, CINAHL, EMBASE and Cochrane Library) and three Chinese databases (CNKI, Wanfang Database, Sinomed) to identify published studies on PTSD prevalence among PLWH from January 2000 to November 2019. The search terms were: posttrauma*, post trauma*, post-trauma*, stress disorder*, OR PTSD; AND HIV, acquired immunodeficiency syndrome, AIDS, OR PLWH\$; AND epidemiology, occurrence, incidence, OR prevalence (see online supplementary appendix 1 for the precise search strategy). The reference lists of review articles and retrieved fulltext articles were also examined for additional papers that were eligible for this review.

\section{Eligibility criteria}

Studies were included for this meta-analysis if they met the following predetermined selection criteria: (1) published in Chinese or English in a peer-reviewed journal; (2) observational study where prevalence figures for PTSD were stated or can be calculated in an adult HIV population and (3) underlined that PTSD cases were identified in accordance with the Diagnostic and Statistical Manual of Mental Disorders (DSM) or the International Classification of Diseases (ICD) diagnostic criteria. ${ }^{28} 29$ Studies were excluded if (1) they only recruited those who had mental distress; (2) they specifically targeted only youth under 18 or children affected by HIV. No restriction was applied regarding gender, nationality or sexual orientation. Additionally, articles were reviewed closely, and if repeated data were observed, only the earlier publication with one set of complete data was included.

\section{Data extraction and analysis}

Screening of papers was carried out by three of the authors (CT, JM and XX) following the Preferred Reporting Items for Systematic Reviews and Meta-Analyses (PRISMA) recommendations. CT and XX searched the English papers and JM screened the Chinese language papers. After duplicates were deleted, each investigator read titles and abstracts closely to capture all potential studies. Fulltext articles were obtained and reviewed for all criteria. Any discrepancies were resolved by discussion with the research team. The PRISMA flow chart shows the results of the systematic search (figure 1).

\section{Data extraction}

Two authors (CT and XX) used a data extraction sheet to capture the following data independently from included papers: first author, year of publication, country of study, sampling method, sample size, number of patients with PTSD, measurements and outcome data. The third investigator (HW) helped to verify all extracted data and resolve any uncertainties. To facilitate detection of sample/ methodological heterogeneity, countries were categorised according to their income level, and measurements 


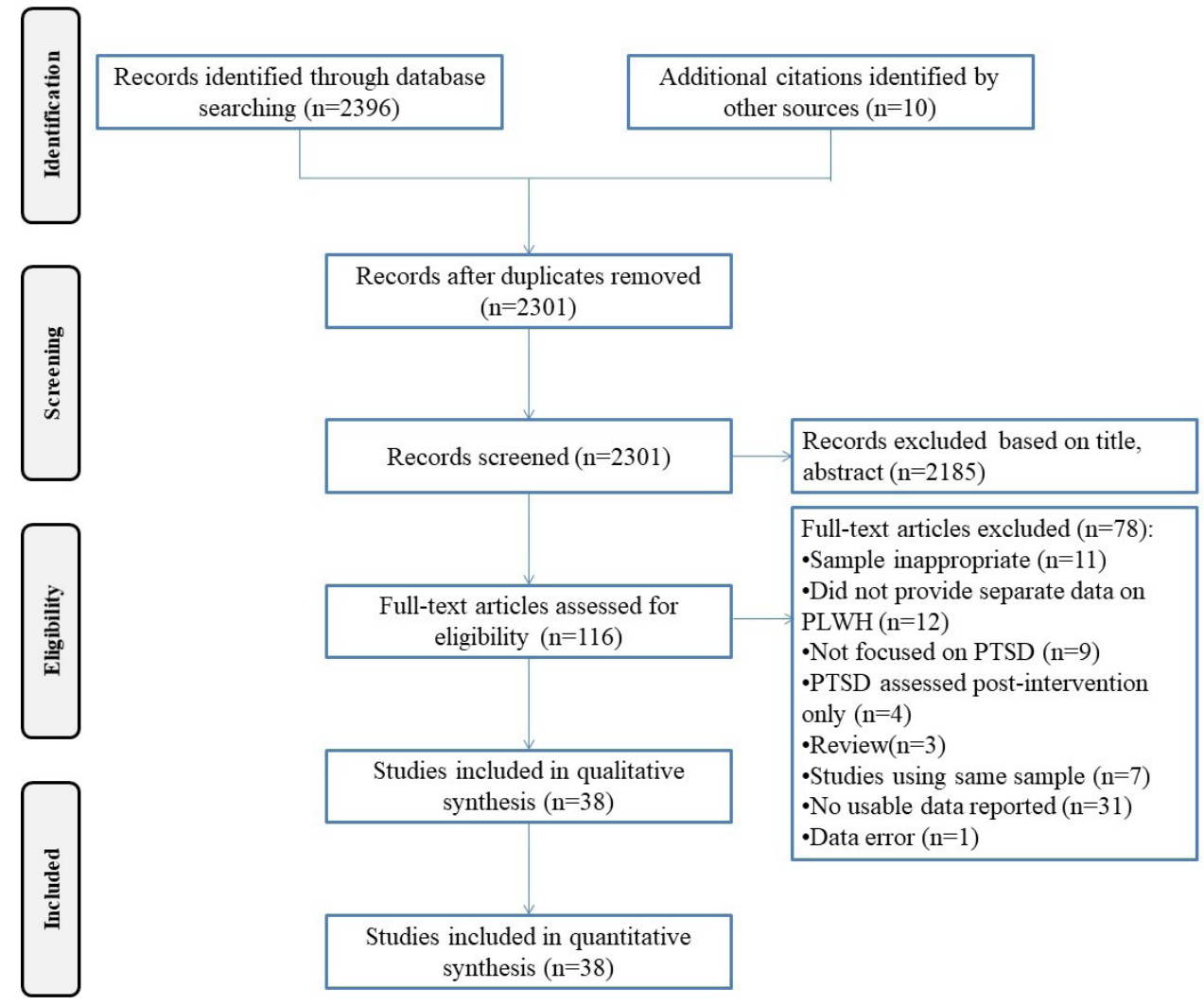

Figure 1 Flow chart of study inclusion. PLWH, people living with HIV; PTSD, post-traumatic stress disorder.

were classified into diagnostic questionnaire and clinical interview based on the approach to diagnosing PTSD. To minimise selection bias, we reviewed the articles included in the meta-analysis to ensure they met eligibility criteria. Gender and sexual orientation were considered because of underlying differences in the epidemics of HIV and trauma. ${ }^{30-32}$ Specifically, populations were grouped as women and men, and samples of HIV-infected men were further classified into men who have sex with men (MSM) and non-MSM males.

\section{Assessment of methodological quality}

We used the quality assessment forms for cross-sectional/ prevalence studies recommended by the US Agency for Healthcare Research and Quality (AHRQ) ${ }^{33}$ to assess the reliability of the eligible studies. The checklist included 11 items. Each item was coded with a 'yes/no/unclear': 'no' or 'unclear' was scored ' 0 ', and 'yes' was scored ' 1 '. Consistent with previous meta-analysis studies using the AHRQ assessments, a total score of $8-11,4-7$ and $0-3$ indicated high, moderate and low quality, respectively.

\section{Meta-analysis}

In this study, all statistical analyses were performed using Stata V.12.0 software (STATA, College Station, Texas, USA). ${ }^{34}$ Heterogeneity was tested and quantified by the $\chi^{2} \mathrm{Q}$ statistic and the $\mathrm{I}^{2}$ statistic. The prevalence of PTSD among PLWH were combined and reported as proportions with corresponding 95\% CIs. The pooled prevalence was estimated using a random-effects model when heterogeneity was statistically significant $\left(\mathrm{I}^{2}>50 \%\right.$, $\mathrm{p} \leq 0.10)$. Otherwise, a fix-effects model was used. ${ }^{35} \mathrm{As}$ sources of heterogeneity may arise from sociodemographic and methodological factors, subgroup analyses were performed by (1) economic levels of the study country, (2) gender/sexual orientation, (3) sampling method and (4) assessment method for PTSD, when enough data were available; $\chi^{2}$ tests were further used to investigate whether there were significant differences between groups. We only performed these subgroup analyses when data were extractable and sufficient. Separate sensitivity analyses were used for studies with low quality to examine the stability of the pooled prevalence. Publication bias was assessed using the Egger's and the Begg's tests. ${ }^{36}{ }^{37}$ Significance level of $p$ values $<0.05$ were employed for all analyses.

\section{Patient and public involvement}

Patients and the public were not involved in this study.

\section{RESULTS}

\section{Search results}

A total of 2396 records were identified by the electronic bibliographic database searches. Ten additional manuscripts were identified through other sources (eg, reference lists of review papers). From an initial screen of 2301 records after duplicates were removed, 2185 records were excluded on basis of title or abstract because those studies were not relevant to this review $(\mathrm{n}=2107)$ or were 
reviews/commentary $(\mathrm{n}=78)$. The remaining 116 full articles were selected for the eligibility assessment. Of those, 7 were excluded for repeated data, 9 were excluded for targeting PLWH who received psychological intervention or suffered from genocide/natural disaster and 62 were excluded for not reporting the PTSD prevalence among PLWH. Finally, a total of 38 studies $^{142425}{ }^{38-72}$ met inclusion criteria and were included in this meta-analysis. Search results are elaborated in figure 1 .

\section{Study characteristics}

Most of the papers (84.3\%) scored 4 or more according to the AHRQ scale indicating a generally moderate to high level of data quality (online supplementary appendix 2 ). Table 1 shows the characteristics of the included studies. These studies were published between $2002^{72}$ and $2019{ }^{38}$ Geographically, over half $(n=21,55.3 \%)$ of the 38 included studies were conducted in the USA, followed by South Africa $(\mathrm{n}=6,15.8 \%)$, China $(\mathrm{n}=3,7.9 \%)$, the UK $(\mathrm{n}=2,5.3 \%)$, Rwanda $(\mathrm{n}=1,2.6 \%)$ and low-income countries including Gambia $(n=1,2.6 \%)$, Nigeria $(n=1,2.6 \%)$, Uganda $(n=1,2.6 \%)$, Haiti $(n=1,2.6 \%)$ and Zimbabwe $(\mathrm{n}=1,2.6 \%)$. Twenty-five studies $(65.8 \%)$ measured PTSD using diagnostic self-report questionnaires, while the other 13 studies $(34.2 \%)$ conducted clinical interviews to diagnose PTSD. Though eight studies $(21.1 \%)$ did not report their sampling methods, convenience sampling methods were adopted in most studies $(n=20,52.6 \%)$, followed by consecutive sampling $(\mathrm{n}=7,18.4 \%)$, and probability sampling $(\mathrm{n}=3,7.9 \%)$. In all, 11743 PLWH were involved (sample size varied from 41 to 1489), of which 2742 were identified with PTSD.

\section{Prevalence of PTSD in PLWH}

The reported prevalence of PTSD in PLWH ranged from $1.6 \%{ }^{57}$ to $54.1 \% .^{59}$ The overall pooled prevalence was $28 \%$ (95\% CI $24 \%$ to $33 \%$ ), estimated by a random-effects model, as significant heterogeneity $\left(\mathrm{p} \leq 0.001, \mathrm{I}^{2}=98.1 \%\right)$ was observed among the included studies. Meta-analyses are shown as forest plots (figure 2).

\section{Publication bias and sensitivity analysis}

Possible publication bias was indicated according to the results of Egger's linear test $(\mathrm{t}=6.84, \mathrm{p}<0.05)$ and Begg's rank test $(\mathrm{z}=0.58, \mathrm{p}=0.56)$. However, low sensitivity was suggested with the pooled prevalence of PTSD changed slightly from $28 \%$ (95\% CI $24 \%$ to $33 \%$ ) to $27 \%$ (95\% CI $22 \%$ to $32 \%$ ) after excluding low-quality articles.

\section{Subgroup analyses}

The results of subgroup analyses are shown in table 2 . The prevalence of PTSD in PLWH differed significantly by geographic area, population group, sampling and measurement method. Specifically, the proportion of PLWH with PTSD in high-income countries tended to be higher (pooled rate $=29 \%, 95 \%$ CI $25 \%$ to $34 \%$ ) than middle-income countries (pooled rate $=27 \%, 95 \%$ CI $16 \%$ to $37 \%$ ) and low-income countries (pooled rate $=26 \%$, $95 \%$ CI $7 \%$ to $45 \%$ ). Studies using clinical interviews reported lower rates of PTSD (pooled rate $=22 \%$, 95\% CI $16 \%$ to $27 \%$ ) compared with those using self-report questionnaires (pooled rate $=31 \%, 95 \%$ CI $27 \%$ to $36 \%$ ). The pooled PTSD prevalence among women living with HIV, MSM living with HIV and non-MSM men living with HIV were $35 \%$ (95\% CI $28 \%$ to $43 \%$ ), $33 \%$ (95\% CI $29 \%$ to $38 \%$ ) and $20 \%$ (95\% CI $17 \%$ to $23 \%$ ), respectively. Also, studies conducted with convenience sampling were more likely to have higher PTSD prevalence (pooled rate $=32 \%$, 95\% CI $27 \%$ to $37 \%$ ) than those using consecutive sampling (pooled rate $=17 \%, 95 \%$ CI $10 \%$ to $24 \%$ ).

\section{DISCUSSION}

To the best of our knowledge, this is the first meta-analysis to provide the pooled estimated prevalence of PTSD among PLWH. A comprehensive search of the literature assessing the rate of comorbidity between PTSD and HIV-infection was performed, and 38 studies met the predefined criteria and were included in the metaanalysis. A total of 11743 participants were involved, of which 2742 were identified with PTSD. The estimated pooled prevalence was $28 \%$ (95\% CI $24 \%$ to $33 \%$ ), which suggests a considerable burden of PTSD in the HIVinfected population. High heterogeneity across studies was detected, and factors such as differences in region, population characteristics, sampling and measurement were associated with the prevalence.

As indicated, the PTSD prevalence among PLWH found in this study (28\%) far exceeded that among the general population $(3.9 \%),{ }^{73}$ and was much higher than the prevalence of PTSD among other vulnerable groups in some previous studies. For example, the prevalence for cancerrelated PTSD was $5.1 \%-15.3 \%^{74}$; in persons with chronic pain, the estimated mean prevalence of PTSD was $9.7 \%{ }^{75}$ The pooled prevalence of PTSD among PLWH found in this study heightened the high rate of comorbidity between HIV infection and PTSD, which is consistent with previous demonstrations. ${ }^{876}$ For PLWH, although depressive symptoms have been the focus of many studies, and $36 \%$ of PLWH were likely to have depression according to nationally representative data in the $\mathrm{USA},{ }^{77}$ this pooled estimated prevalence of PTSD among PLWH showed that PTSD is also a common mental disorder among individuals living with HIV/AIDS. Therefore, the results of our study significantly underscore the importance of early assessment and trauma-directed psychological interventions for PLWH.

Undoubtedly, heterogeneity between studies is expected in such a relatively large topic of review. In this review, the estimated PTSD prevalence in PLWH varied widely $(1.6 \%-54.1 \%)$ across studies and countries, which is also a common problem in the epidemiology of psychological and mental health disorders. ${ }^{78}$ Consistent with the findings of previous research, ${ }^{59}$ the majority of studies which have determined the incidence of HIV-related PTSD have been conducted in high-income countries $(n=23)$. Compared with low-income countries, high-income 
Table 1 Characteristics of the included studies

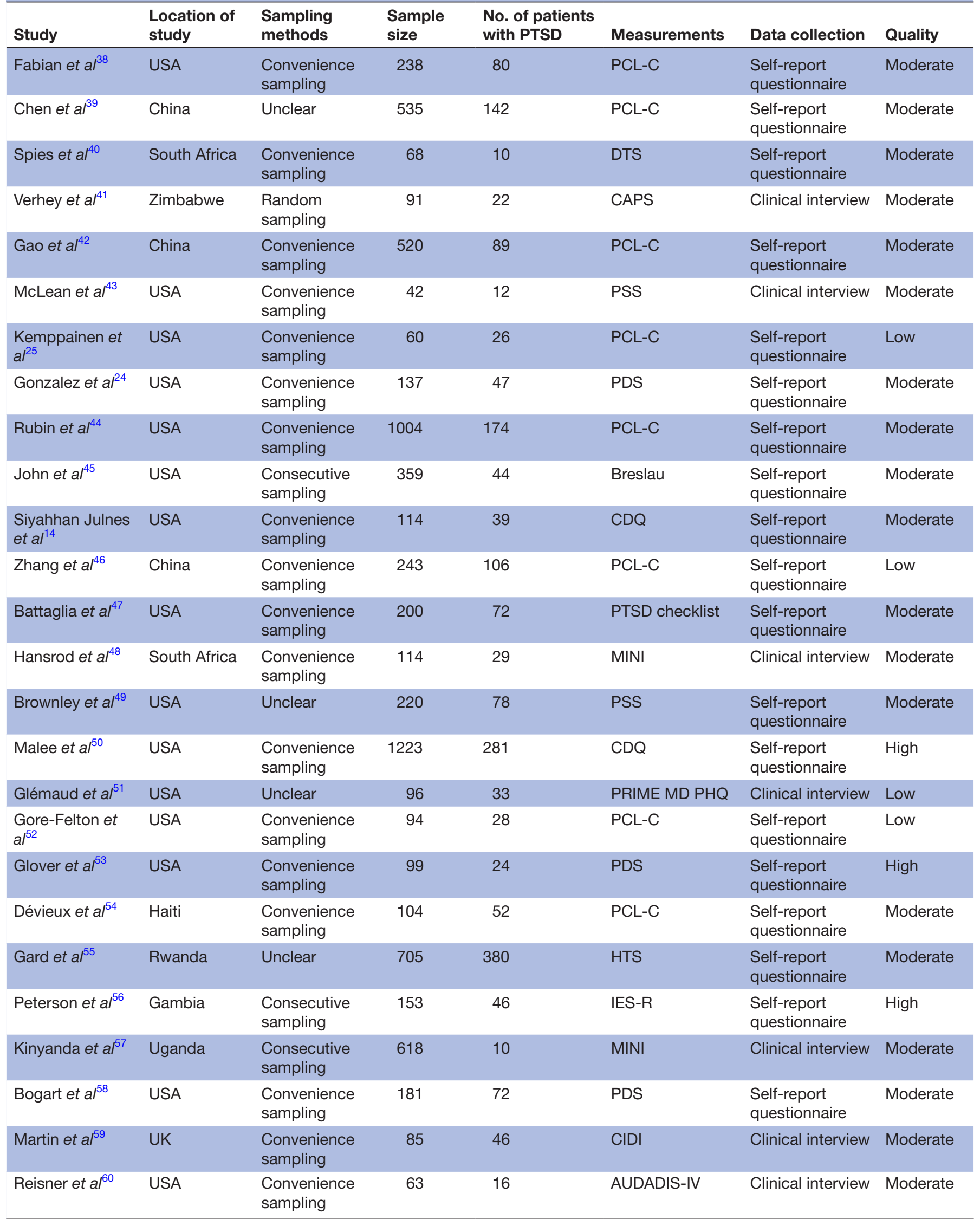




\begin{tabular}{|c|c|c|c|c|c|c|c|}
\hline Study & $\begin{array}{l}\text { Location of } \\
\text { study }\end{array}$ & $\begin{array}{l}\text { Sampling } \\
\text { methods }\end{array}$ & $\begin{array}{l}\text { Sample } \\
\text { size }\end{array}$ & $\begin{array}{l}\text { No. of patients } \\
\text { with PTSD }\end{array}$ & Measurements & Data collection & Quality \\
\hline Joska et al ${ }^{62}$ & South Africa & Unclear & 536 & 164 & HTS & $\begin{array}{l}\text { Self-report } \\
\text { questionnaire }\end{array}$ & Moderate \\
\hline Spies et $a l^{64}$ & South Africa & Unclear & 429 & 92 & MINI & Clinical interview & Moderate \\
\hline Fincham et $a l^{65}$ & South Africa & $\begin{array}{l}\text { Consecutive } \\
\text { sampling }\end{array}$ & 456 & 23 & MINI & Clinical interview & Moderate \\
\hline Zanjani et al ${ }^{66}$ & USA & $\begin{array}{l}\text { Random } \\
\text { sampling }\end{array}$ & 109 & 13 & MINI & Clinical interview & Moderate \\
\hline Leserman et $a l^{69}$ & USA & $\begin{array}{l}\text { Consecutive } \\
\text { sampling }\end{array}$ & 611 & 98 & PTSD checklist & $\begin{array}{l}\text { Self-report } \\
\text { questionnaire }\end{array}$ & High \\
\hline Olley et $a l^{70}$ & South Africa & $\begin{array}{l}\text { Consecutive } \\
\text { sampling }\end{array}$ & 149 & 42 & MINI & Clinical interview & Moderate \\
\hline Vitiello et $a^{71}$ & USA & $\begin{array}{l}\text { Probability } \\
\text { sample }\end{array}$ & 1489 & 155 & CIDI & Clinical interview & Moderate \\
\hline Martinez et $a l^{72}$ & USA & $\begin{array}{l}\text { Consecutive } \\
\text { sampling }\end{array}$ & 41 & 17 & PCL-C & $\begin{array}{l}\text { Self-report } \\
\text { questionnaire }\end{array}$ & Moderate \\
\hline
\end{tabular}

*Total number of adults living with HIV.

AUDADIS-IV, The Alcohol Use Disorder and Associated Disabilities Interview Schedule-IV; CAPS, Clinician Administered Post-traumatic Stress scale; CDQ, Client Diagnostic Questionnaire; CIDI, Clinical Interview for Diagnosis for DSM-IV; DIS-IV, Diagnostic Interview Schedule for DSM-IV diagnoses; DTS, Davidson Trauma Scale; HTS, Harvard Trauma Scale; IES-R, Impact of Event Scale-Revised; MINI, Mini-International Neuropsychiatric Interview; PCL-C, Post-traumatic Stress Disorder Checklist-Civilian Version; PDS, Posttraumatic Stress Diagnostic Scale-Self-report version; PRIME MD PHQ, Primary Care Evaluation of Mental Disorders Patient Health Questionnaire; PSS, Post-traumatic Stress Symptom Scale; PTSD checklist, post-traumatic stress disorder checklist.

countries reported relatively higher prevalence of PTSD. This tendency and the varied prevalence of PTSD among PLWH may be explained by the cross-cultural differences in attitudes towards HIV infection. Turan $e t a l^{80}$ found that the level of perceived HIV-related stigma in the community experienced by PLWH may cause adverse health and psychosocial outcomes. Social isolation and stigma might exacerbate symptoms of PTSD. ${ }^{81}$ In high-income countries, PLWH may experience 'compound' or 'layered' stigma, where stigmatising beliefs are associated with HIV infection and related to sexual orientation, commercial sexual behaviours, etc. ${ }^{82}$ Although, HIV-related stigma is highly burdensome in some middle-income countries, such as China, ${ }^{8384}$ the reported prevalence of PLWH with PTSD was lower compared with high-income countries. The differences between collectivism and individualism rooted in eastern and western culture may be an explanation. ${ }^{85}$ It is worth noting that social context may influence the susceptibility of mental health disorders, and constitutes an important factor in perceived stigma among individuals with mental illness. Compared with high-income countries, stigmatising beliefs about the causes and presence of mental illness are still widespread in low-income and middle-income countries ${ }^{86}{ }^{87}$ which may lead to less disease reporting. Caution must be applied, since there is a lack of studies investigating HIV-related PTSD in lowincome $(n=5)$ and middle-income $(n=10)$ countries. In particular, detection of prevalence is associated with the representativeness and size of sample. In the context of low-income and middle-income countries, it could be argued that lower HIV testing rates may underestimate the PTSD prevalence and impact generalisability. Thus, more epidemiological studies are warranted to better understand and clarify the difference in PTSD prevalence among PLWH globally.

Subgroup analyses indicated that the prevalence of PTSD differed significantly when gender and sexual orientation were taken into consideration. Specifically, women living with HIV exhibited a higher prevalence of PTSD (35\%) in comparison with both MSM (33\%) and non-MSM male (20\%) groups. This matches the pattern of prevalence in PTSD, in which being female is a risk factor associated with PTSD development. ${ }^{16}$ As suggested by the aforementioned findings, MSM living with HIV may experience higher levels of stigma and trauma related to their sexual orientation. ${ }^{30} 88$ Compared with 


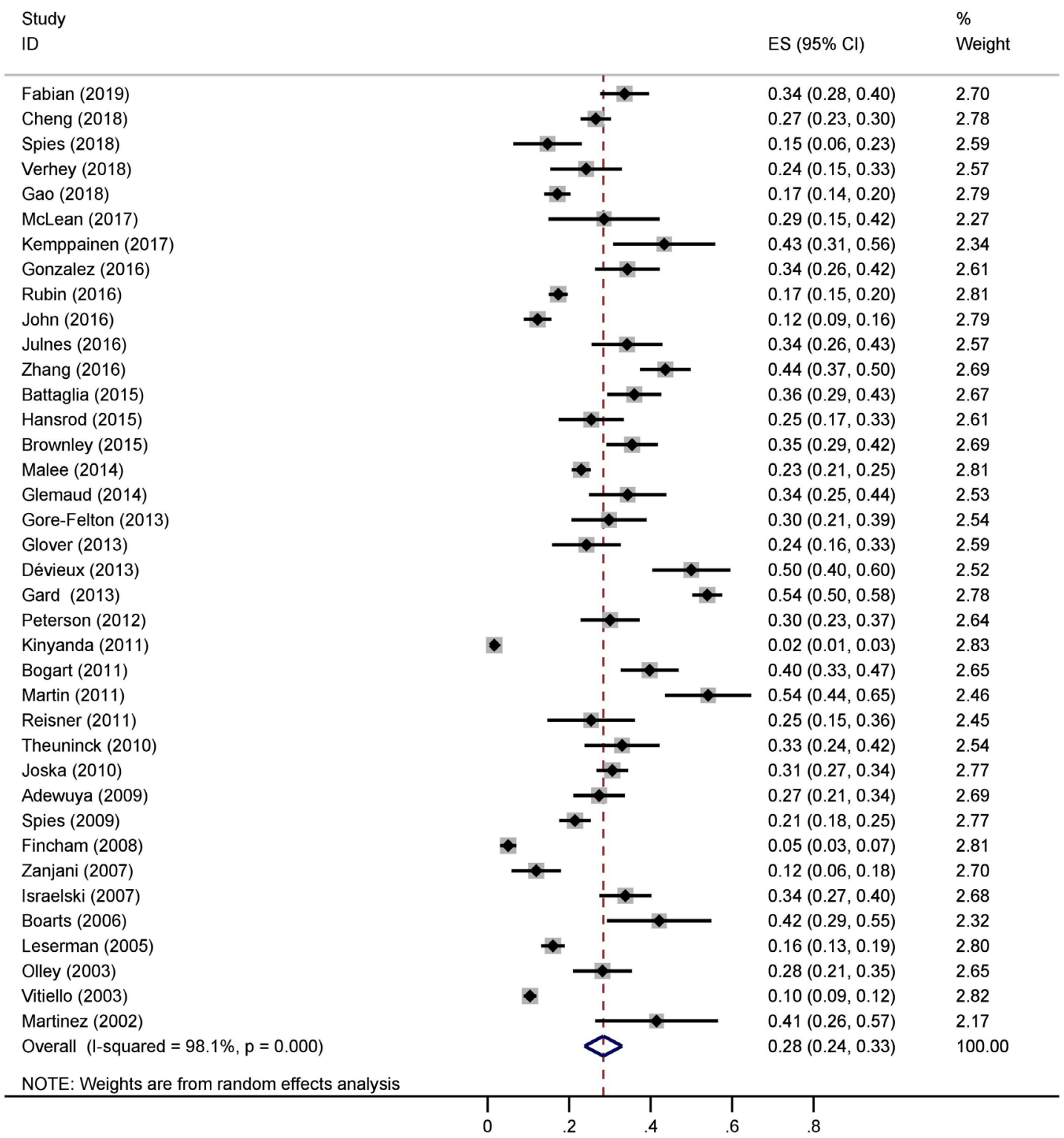

Figure 2 Forest plot presenting the prevalence of post-traumatic stress disorder (PTSD) in people living with HIV (PLWH).

heterosexual counterparts, MSM reported higher risk for suicidal ideation, ${ }^{89}$ which also indicates greater psychological stress and mental health problems (ie, PTSD and depression). The findings that MSM and female HIVinfected individuals are at higher risk of developing PTSD may indicate that related health promotion campaigns should be directed to these groups. Clinicians should also take into consideration the PTSD susceptibility in different groups of HIV-infected populations during diagnoses. Notably, most eligible studies did not provide separate prevalence regarding the gender of participants, and only three studies identified PTSD prevalence among MSM living with HIV. Given that these data are from a small number of studies and/or participants, this finding should be interpreted with caution. Nonetheless, given the high prevalence of PTSD in this group, future efforts should be made to obtain more prevalence data on PTSD among MSM living with HIV.
In our study, we assessed methodological differences that may result in heterogeneity. Factors including sampling strategies (convenience vs consecutive) and measurement (self-report vs clinical interview) were evaluated by subgroup analyses. Among 38 studies providing estimated prevalence of PTSD, 7 reported participants were consecutively enrolled via self-referral from flyers describing the study or advertisements from HIV healthcare providers, and generated a lower pooled rate $(17 \%)$ compared with the other 20 studies using convenience sampling (32\%), although both sampling methods were non-probabilistic. An increase in reporting rate due to convenience sampling has also been found in another meta-analysis synthesising the HCV seroconversion rates in MSM living with HIV. ${ }^{90}$ Convenience samples may allow researchers to select participants who are more likely to have PTSD symptoms and lead to overestimation, but given that only four studies were rated as high 


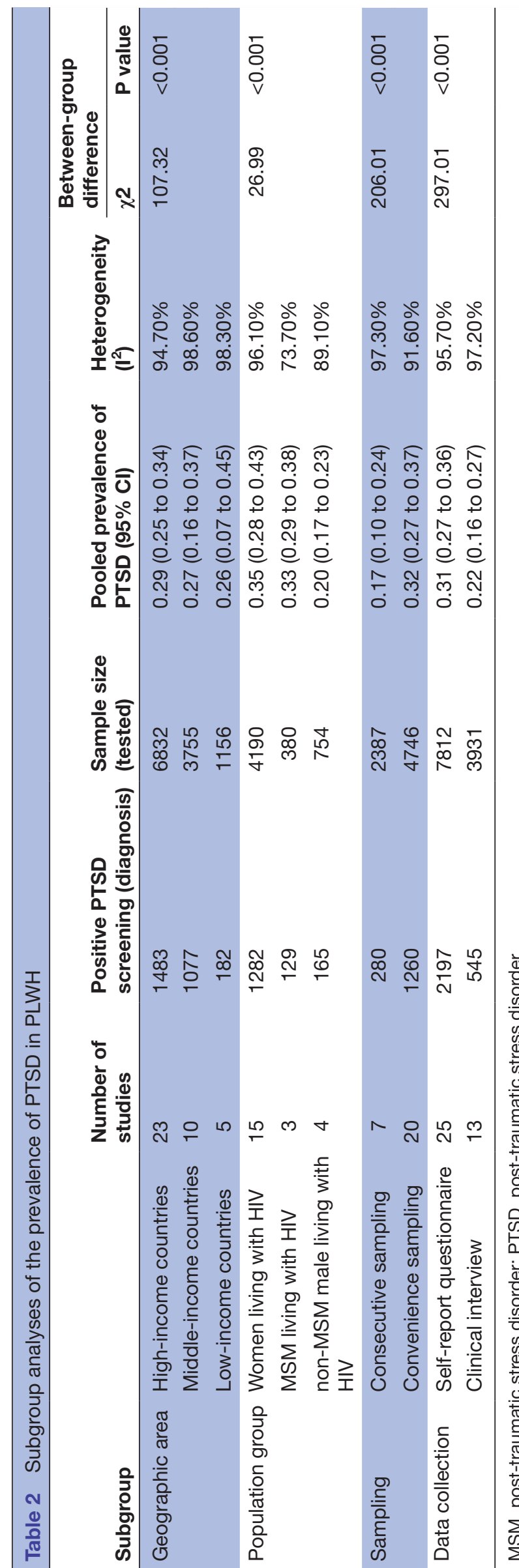

quality and limited description of study methods was detected in some studies, the reporting of epidemiology may not be sufficient to draw conclusions. Therefore, the appropriateness of calculating pooled prevalence estimates could be questioned, and the pooled prevalence estimate regarding the varied recruitment approaches of the studies should be interpreted with caution.

In this review, a total of 14 different tools were used to assess PTSD symptoms among PLWH across studies. Overall, the prevalence of PTSD screened by self-report instruments $(31 \%)$ was higher than that assessed by clinical interview (22\%). Although both measurements were in accordance with the diagnostic criteria (ie, DSM or ICD), clinical interviews most likely do a much better job of assessing symptoms carefully-for example, not counting symptoms that do not meet clinically meaningful thresholds, and self-report measures may inflate the prevalence estimates ${ }^{91}$; alternatively, subjects may be more comfortable reporting symptoms using self-report questionnaires (presumably paper or online surveys rather than face-to-face interviews). Screening or diagnosing with survey instruments is a common and practical method, especially in resource-limited regions. We found that, although a number of measures have been used to assess PTSD among PLWH, there is no tool designed for this population specifically. As the sensitivity and specificity of the measures may impact accurate PTSD detection, further research is required to identify a standardised measure accordingly to improve validity.

Certain limitations should be acknowledged in this meta-analysis. First, although minimal potential for publication bias was presumed as all data drawn for this review were from studies reporting estimated point prevalence without intervention, publication bias cannot be ruled out for there are a number of studies published in languages other than English and Chinese that were not included in this review. Second, the heterogeneity was high in the whole sample and most subgroups. Heterogeneity importantly influences the reliability and accuracy of a meta-analysis, and the only reliable method for acquiring correct inferences is careful selection of appropriate studies. ${ }^{92}$ Even though strict eligibility criteria were employed in addressing PTSD prevalence in this study, it should be noted that getting HIV is only one possible traumatic event, and therefore it is possible that the PTSD experienced by participants in the selected studies was due to an event other than finding out they were HIV positive. Accordingly, we suggest that further studies assessing PTSD among PLWH include more detail on the specific traumas experienced by study participants. In addition, we have analysed the influences of region, population group, recruitment venue and measurement method, which explained some of the heterogeneity. However, the results should be interpreted with caution. Third, although some studies have indicated that several sociodemographic factors such as age may be associated with PTSD incidence among PLWH, we were unable to conduct subgroup analyses stratified by age due to the 
lack of detailed information reported in the published studies. As the quality of reporting could increase the susceptibility to bias, it is imperative for researchers to report their findings of observational studies in accordance with the appropriate guidelines, such as the Strengthening the Reporting of Observational Studies in Epidemiology checklist.

\section{CONCLUSIONS}

This review contributes to evidence on the quantitatively pooled prevalence of PTSD among PLWH. Stratified subgroups including geographic area, population group, sampling and measurement method were found to be significant factors accounting for the varied prevalence of PTSD among PLWH. More effective intervention strategies targeting PTSD among PLWH are urgently needed. Future efforts should be made to obtain countries' own PTSD prevalence data among PLWH as well as specific vulnerable minorities, which will provide a basis for public health policy, healthcare planning and resource allocation for PTSD intervention initiatives. Our study also suggests that more research using robust and comprehensive methodology is needed to provide rigorous evidence for designing the targeted psychological interventions.

Acknowledgements The authors would like to thank Dr Tian Hongping, Rory Meyers College of Nursing, New York University for assistance in this systematic review.

Contributors Conception of the work: HW and CT. Systematic review and article evaluation: CT, JM, XX and LZ. Data analysis: CT and XX. Results interpretation: CT, LAG, HW and ABW. Drafting the article: CT, LAG, HW and ABW. Critical revision of the manuscript: LAG, HW and ABW. Final approval of the manuscript: all the authors. All the authors fulfil the ICMJE criteria for authorship.

Funding CT is supported by the Scholarship from China Scholarship Council (CSC.201806370166).

Competing interests None declared.

Patient and public involvement Patients and/or the public were not involved in the design, conduct, reporting or dissemination plans of this research.

Patient consent for publication Not required.

Ethics approval As this study is a systematic review based on published studies, ethical approval is not required.

Provenance and peer review Not commissioned; externally peer reviewed.

Data availability statement All data relevant to the study are included in the article or uploaded as supplementary information.

Open access This is an open access article distributed in accordance with the Creative Commons Attribution Non Commercial (CC BY-NC 4.0) license, which permits others to distribute, remix, adapt, build upon this work non-commercially, and license their derivative works on different terms, provided the original work is properly cited, appropriate credit is given, any changes made indicated, and the use is non-commercial. See: http://creativecommons.org/licenses/by-nc/4.0/.

ORCID iD

Chulei Tang http://orcid.org/0000-0003-3178-5130

\section{REFERENCES}

1 UNAIDS. UNAIDS data, 2018. Available: http://www.unaids.org/en/ resources/documents/2018/unaids-data-2018

2 Niu L, Luo D, Liu Y, et al. The mental health of people living with HIV in China, 1998-2014: a systematic review. PLoS One 2016;11:e0153489.
3 Adams C, Zacharia S, Masters L, et al. Mental health problems in people living with HIV: changes in the last two decades: the London experience 1990-2014. AIDS Care 2016;28(Suppl 1):56-9.

4 Friedman MJ, Resick PA, Bryant RA, et al. Considering PTSD for DSM-5. Depress Anxiety 2011;28:750-69.

5 Young C. Understanding HIV-related posttraumatic stress disorder in South Africa: a review and conceptual framework. Afr J AIDS Res 2011;10:139-48.

6 Sherr L, Nagra N, Kulubya G, et al. HIV infection associated posttraumatic stress disorder and post-traumatic growth--a systematic review. Psychol Health Med 2011;16:612-29.

7 Cavanaugh CE, Hansen NB, Sullivan TP. Hiv sexual risk behavior among low-income women experiencing intimate partner violence: the role of posttraumatic stress disorder. AIDS Behav 2010;14:318-27.

8 Neigh GN, Rhodes ST, Valdez A, et al. Ptsd co-morbid with HIV: separate but equal, or two parts of a whole? Neurobiol Dis 2016;92:116-23.

9 Borwein A, Salters KA, Palmer AK, et al. High rates of lifetime and recent violence observed among harder-to-reach women living with HIV. AIDS Care 2014;26:587-94.

10 Luo S, Lin C, Ji G, et al. Post-Traumatic stress disorder symptoms among people living with HIV/AIDS in rural China. AIDS Behav 2017;21:3202-8.

11 Kessler RC. Posttraumatic stress disorder: the burden to the individual and to Society. J Clin Psychiatry 2000;61(Suppl 5):4-14.

12 Pence BW. The impact of mental health and traumatic life experiences on antiretroviral treatment outcomes for people living with HIV/AIDS. J Antimicrob Chemother 2009;63:636-40.

13 Ebrahimzadeh Z, Goodarzi MA, Joulaei H. Predicting the antiretroviral medication adherence and CD4 measure in patients with HIV/AIDS based on the post traumatic stress disorder and depression. Iran $J$ Public Health 2019;48:139-46.

14 Siyahhan Julnes P, Auh S, Krakora R, et al. The association between post-traumatic stress disorder and markers of inflammation and immune activation in HIV-infected individuals with controlled viremia. Psychosomatics 2016;57:423-30.

15 Lynn C, Chenneville T, Bradley-Klug K, et al. Depression, anxiety, and posttraumatic stress as predictors of immune functioning: differences between youth with behaviorally and perinatally acquired HIV. AIDS Care 2019;31:1261-70.

16 Brief DJ, Bollinger AR, Vielhauer MJ, et al. Understanding the interface of HIV, trauma, post-traumatic stress disorder, and substance use and its implications for health outcomes. AIDS Care 2004;16(Suppl 1):97-120.

17 Nightingale VR, Sher TG, Mattson M, et al. The effects of traumatic stressors and HIV-related trauma symptoms on health and health related quality of life. AIDS Behav 2011;15:1870-8.

18 Koegler E, Kennedy CE. A scoping review of the associations between mental health and factors related to HIV acquisition and disease progression in conflict-affected populations. Confl Health 2018;12:20.

19 Pearson CR, Kaysen D, Huh D, et al. Randomized control trial of culturally adapted cognitive processing therapy for PTSD substance misuse and HIV sexual risk behavior for native American women. AIDS Behav 2019;23:695-706.

20 Tull MT, Weiss NH, McDermott MJ. Post-traumatic stress disorder and impulsive and risky behavior: overview and discussion of potential mechanisms. In: Comprehensive guide to post-traumatic stress disorders. . Springer, Cham, 2016: 2. 803-16.

21 Weiss NH, Tull MT, Borne MER, et al. Posttraumatic stress disorder symptom severity and HIV-risk behaviors among substancedependent inpatients. AIDS Care 2013;25:1219-26.

22 Applebaum AJ, Bedoya CA, Hendriksen ES, et al. Future directions for interventions targeting PTSD in HIV-infected adults. J Assoc Nurses AIDS Care 2015;26:127-38.

23 Machtinger EL, Wilson TC, Haberer JE, et al. Psychological trauma and PTSD in HIV-positive women: a meta-analysis. AIDS Behav 2012;16:2091-100.

24 Gonzalez A, Locicero B, Mahaffey B, et al. Internalized HIV stigma and mindfulness: associations with PTSD symptom severity in trauma-exposed adults with HIV/AIDS. Behav Modif 2016;40:144-63.

25 Kemppainen JK, MacKain S, Alexander M, et al. Posttraumatic stress disorder and stressful life events among rural women with HIV disease. J Assoc Nurses AIDS Care 2017;28:216-25.

26 Rubin LH, Cook JA, Springer G, et al. Perceived and post-traumatic stress are associated with decreased learning, memory, and fluency in HIV-infected women. AIDS 2017;31:2393-401.

27 Seedat S. Interventions to improve psychological functioning and health outcomes of HIV-infected individuals with a history of trauma or PTSD. Curr HIVIAIDS Rep 2012;9:344-50. 
28 World Health Organization. The ICD-10 classification of mental and behavioural disorders: clinical descriptions and diagnostic guidelines. Geneva: World Health Organization, 1992.

29 American Psychiatric Pub. Diagnostic and statistical manual of mental disorders. 5th edn. Washington, DC: American Psychiatric Pub, 2013.

30 Sales JM, Swartzendruber A, Phillips AL. Trauma-Informed HIV prevention and treatment. Curr HIVIAIDS Rep 2016;13:374-82.

31 Burnham KE, Cruess DG, Kalichman MO, et al. Trauma symptoms, internalized stigma, social support, and sexual risk behavior among HIV-positive gay and bisexual MSM who have sought sex partners online. AIDS Care 2016;28:347-53.

32 Rzeszutek M, Oniszczenko W, Firląg-Burkacka E. Gender differences in posttraumatic stress symptoms and social support in a sample of HIV-positive individuals. Women Health 2017;57:792-803

33 Rostom A, Dubé C, Cranney A. Appendix D quality assessment forms. Agency for Healthcare Research and Quality, 2004. https:// www.ncbi.nlm.nih.gov/books/NBK35156/

34 Stata C, Station C. TX. Stata press, 2016

35 Higgins JPT, Thompson SG. Quantifying heterogeneity in a metaanalysis. Stat Med 2002;21:1539-58.

36 Egger M, Davey Smith G, Schneider M, et al. Bias in meta-analysis detected by a simple, graphical test. BMJ 1997;315:629-34.

37 Begg CB, Mazumdar M. Operating characteristics of a RANK correlation test for publication bias. Biometrics 1994;50:1088-101.

38 Fabian KE, Huh D, Kemp CG, et al. Moderating factors in an Antistigma intervention for African American women with HIV in the United States: a secondary analysis of the unity trial. AIDS Behav 2019;23:2432-42.

39 Chen CD, Yan H, SY L, et al. Post-Traumatic stress disorder and it associated factors among people living with HIV in Wuhan City. Chin $J$ Public Health 2018;21:1-4.

40 Spies G, Konkiewitz EC, Seedat S. Incidence and persistence of depression among women living with and without HIV in South Africa: a longitudinal study. AIDS Behav 2018;22:3155-65.

41 Verhey R, Chibanda D, Gibson L, et al. Validation of the posttraumatic stress disorder checklist - 5 (PCL-5) in a primary care population with high HIV prevalence in Zimbabwe. BMC Psychiatry 2018;18:109.

42 Gao M, Xiao C, Zhang X, et al. Social capital and PTSD among PLWHA in China: the mediating role of resilience and internalized stigma. Psychol Health Med 2018;23:698-706.

43 McLean CP, Gay NG, Metzger DA, et al. Psychiatric symptoms and barriers to care in HIV-infected individuals who are lost to care. $J$ Int Assoc Provid AIDS Care 2017;16:423-9.

44 Rubin LH, Pyra M, Cook JA, et al. Post-Traumatic stress is associated with verbal learning, memory, and psychomotor speed in HIV-infected and HIV-uninfected women. J Neurovirol 2016;22:159-69.

45 John MD, Greene M, Hessol NA, et al. Geriatric assessments and association with VACS index among HIV-infected older adults in San Francisco. J Acquir Immune Defic Syndr 2016;72:534-41.

46 Zhang X, Wang T, Liu M, et al. Post-Traumatic stress disorder among people living with HIV/AIDS and its influence factors. Chongqing Medicine 2016:232-5

47 Battaglia TA, Gunn CM, McCoy ME, et al. Beliefs about anal cancer among HIV-infected women: barriers and motivators to participation in research. Womens Health Issues 2015;25:720-6.

48 Hansrod F, Spies G, Seedat S. Type and severity of intimate partner violence and its relationship with PTSD in HIV-infected women. Psychol Health Med 2015;20:697-709.

49 Brownley JR, Fallot RD, Wolfson Berley R, et al. Trauma history in African-American women living with HIV: effects on psychiatric symptom severity and religious coping. AIDS Care 2015;27:964-71.

50 Malee KM, Mellins CA, Huo Y, et al. Prevalence, incidence, and persistence of psychiatric and substance use disorders among mothers living with HIV. J Acquir Immune Defic Syndr 2014;65:526-34.

51 Glémaud $\mathrm{M}$, Illa L, Echenique $\mathrm{M}$, et al. Abuse and mental health concerns among HIV-infected Haitian women living in the United States. J Assoc Nurses AIDS Care 2014;25:S62-9.

52 Gore-Felton C, Ginzburg K, Chartier M, et al. Attachment style and coping in relation to posttraumatic stress disorder symptoms among adults living with HIV/AIDS. J Behav Med 2013;36:51-60.

53 Glover DA, Williams JK, Kisler KA. Using novel methods to examine stress among HIV-positive African American men who have sex with men and women. J Behav Med 2013;36:283-94.

54 Dévieux JG, Malow RM, Attonito JM, et al. Post-Traumatic stress disorder symptomatology and alcohol use among HIV-seropositive adults in Haiti. AIDS Care 2013;25:1210-8.
55 Gard TL, Hoover DR, Shi Q, et al. The impact of HIV status, HIV disease progression, and post-traumatic stress symptoms on the health-related quality of life of Rwandan women genocide survivors. Qual Life Res 2013;22:2073-84.

56 Peterson K, Togun T, Klis S, et al. Depression and posttraumatic stress disorder among HIV-infected Gambians on antiretroviral therapy. AIDS Patient Care STDS 2012;26:589-96.

57 Kinyanda E, Hoskins S, Nakku J, et al. Prevalence and risk factors of major depressive disorder in HIV/AIDS as seen in semi-urban Entebbe district, Uganda. BMC Psychiatry 2011;11:205.

58 Bogart LM, Wagner GJ, Galvan FH, et al. Perceived discrimination and mental health symptoms among black men with HIV. Cultur Divers Ethnic Minor Psychol 2011;17:295-302.

59 Martin L, Kagee A, Lifetime KA. Lifetime and HIV-related PTSD among persons recently diagnosed with HIV. AIDS Behav 2011;15:125-31.

60 Reisner SL, Falb KL, Mimiaga MJ. Early life traumatic stressors and the mediating role of PTSD in incident HIV infection among US men, comparisons by sexual orientation and race/ethnicity: results from the NESARC, 2004-2005. J Acquir Immune Defic Syndr 2011;57:340-50.

61 Theuninck AC, Lake N, Gibson S. Hiv-Related posttraumatic stress disorder: investigating the traumatic events. AIDS Patient Care STDS 2010;24:485-91.

62 Joska JA, Fincham DS, Stein DJ, et al. Clinical correlates of HIVassociated neurocognitive disorders in South Africa. AIDS Behav 2010;14:371-8.

63 Adewuya AO, Afolabi MO, Ola BA, et al. Post-Traumatic stress disorder (PTSD) after stigma related events in HIV infected individuals in Nigeria. Soc Psychiatry Psychiatr Epidemiol 2009;44:761-6.

64 Spies G, Kader K, Kidd M, et al. Validity of the K-10 in detecting DSM-IV-defined depression and anxiety disorders among HIVinfected individuals. AIDS Care 2009;21:1163-8.

65 Fincham D, Smit J, Carey P, et al. The relationship between behavioural inhibition, anxiety disorders, depression and CD4 counts in HIV-positive adults: a cross-sectional controlled study. AIDS Care 2008;20:1279-83.

66 Zanjani F, Saboe K, Oslin D. Age difference in rates of mental health/ substance abuse and behavioral care in HIV-positive adults. AIDS Patient Care STDS 2007;21:347-55

67 Israelski DM, Prentiss DE, Lubega S, et al. Psychiatric co-morbidity in vulnerable populations receiving primary care for HIV/AIDS. AIDS Care 2007;19:220-5.

68 Boarts JM, Sledjeski EM, Bogart LM, et al. The differential impact of PTSD and depression on HIV disease markers and adherence to HAART in people living with HIV. AIDS Behav 2006;10:253-61.

69 Leserman J, Whetten K, Lowe K, et al. How trauma, recent stressful events, and PTSD affect functional health status and health utilization in HIV-infected patients in the South. Psychosom Med 2005;67:500-7.

70 Olley BO, Gxamza F, Seedat S, et al. Psychopathology and coping in recently diagnosed HIV/AIDS patients--the role of gender. S Afr Med $J$ 2003;93:928-31.

71 Vitiello B, Burnam MA, Bing EG, et al. Use of psychotropic medications among HIV-infected patients in the United States. Am J Psychiatry 2003;160:547-54.

72 Martinez A, Israelski D, Walker C, et al. Posttraumatic stress disorder in women attending human immunodeficiency virus outpatient clinics. AIDS Patient Care STDS 2002;16:283-91.

73 Koenen KC, Ratanatharathorn A, Ng L, et al. Posttraumatic stress disorder in the world mental health surveys. Psychol Med 2017:47:2260-74.

74 Abbey G, Thompson SBN, Hickish T, et al. A meta-analysis of prevalence rates and Moderating factors for cancer-related posttraumatic stress disorder. Psychooncology 2015;24:371-81.

75 Siqveland J, Hussain A, Lindstrøm JC, et al. Prevalence of posttraumatic stress disorder in persons with chronic pain: a metaanalysis. Front Psychiatry 2017;8:164.

76 Kelly B, Raphael B, Judd F, et al. Posttraumatic stress disorder in response to HIV infection. Gen Hosp Psychiatry 1998;20:345-52.

77 Bing EG, Burnam MA, Longshore D, et al. Psychiatric disorders and drug use among human immunodeficiency virus-infected adults in the United States. Arch Gen Psychiatry 2001:58:721-8.

78 Pringsheim T, Jette N, Frolkis A, et al. The prevalence of Parkinson's disease: a systematic review and meta-analysis. Mov Disord 2014;29:1583-90.

79 Wang T, Fu H, Kaminga AC, et al. Prevalence of depression or depressive symptoms among people living with HIV/AIDS in China: a systematic review and meta-analysis. BMC Psychiatry 2018;18:160.

80 Turan B, Budhwani H, Fazeli PL, et al. How does stigma affect people living with HIV? the mediating roles of internalized and anticipated 
HIV stigma in the effects of perceived community stigma on health and psychosocial outcomes. AIDS Behav 2017;21:283-91.

81 Kamen C, Vorasarun C, Canning T, et al. The impact of stigma and social support on development of post-traumatic growth among persons living with HIV. J Clin Psychol Med Settings 2016;23:126-34

82 Lowther K, Selman L, Harding R, et al. Experience of persistent psychological symptoms and perceived stigma among people with HIV on antiretroviral therapy (art): a systematic review. Int J Nurs Stud 2014;51:1171-89.

83 Li Z, Morano JP, Khoshnood K, et al. HIV-related stigma among people living with HIV/AIDS in rural central China. BMC Health Serv Res 2018;18:453.

84 Li L, Liang L-J, Lin C, et al. HIV prevention intervention to reduce HIV-related stigma: evidence from China. AIDS 2010;24:115-22.

85 Shavitt S, Cho YI, Johnson TP, et al. Culture moderates the relation between perceived stress, social support, and mental and physical health. J Cross Cult Psychol 2016;47:956-80.

86 Lauber C, Rössler W. Stigma towards people with mental illness in developing countries in Asia. Int Rev Psychiatry 2007;19:157-78.
87 Seeman N, Tang S, Brown AD, et al. World survey of mental illness stigma. J Affect Disord 2016;190:115-21.

88 Reisner SL, Mimiaga MJ, Safren SA, et al. Stressful or traumatic life events, post-traumatic stress disorder (PTSD) symptoms, and HIV sexual risk taking among men who have sex with men. AIDS Care 2009;21:1481-9.

89 Luo Z, Feng T, Fu H, et al. Lifetime prevalence of suicidal ideation among men who have sex with men: a meta-analysis. $B M C$ Psychiatry 2017; 17:406.

90 Hagan $\mathrm{H}$, Jordan AE, Neurer $\mathrm{J}$, et al. Incidence of sexually transmitted hepatitis $\mathrm{C}$ virus infection in HIV-positive men who have sex with men. AIDS 2015;29:2335-45.

91 Ayers S, Wright DB, Ford E. Hyperarousal symptoms after traumatic and nontraumatic births. J Reprod Infant Psychol 2015;33:282-93.

92 Melsen WG, Bootsma MCJ, Rovers MM, et al. The effects of clinical and statistical heterogeneity on the predictive values of results from meta-analyses. Clin Microbiol Infect 2014;20:123-9. 